\title{
Effectiveness of outpatient-based group cognitive-behavioral therapy in Japanese patients with mood disorders and neurotic disorders
}

\author{
Norimasa Sawaguchi ${ }^{1}$, Hiroe Onaka ${ }^{1}$, Ryoko Hikima ${ }^{1}$, Akinobu Kuroki ${ }^{1}$, Emiko Hatayama ${ }^{1}$, Hiroshi Yano $^{2}$, \\ Katsunaka Mikami ${ }^{2}$, Kenji Yamamoto ${ }^{2}$
}

${ }^{1}$ Department of Medical Technology, Tokai University Hospital, Isehara, Kanagawa, Japan.

2 Department of Psychiatry, Tokai University School of Medicine, Isehara, Kanagawa, Japan.

Received: 08/15/2017 - Accepted: 09/10/2018

DOl: 10.1590/0101-60830000000169

\begin{abstract}
Background: Although the effectiveness of group cognitive-behavioral therapy (GCBT) has been studied, evidence is still not sufficient. Objective: The aim of the present study is to consider the effectiveness of GCBT with mood disorders and neurotic disorders. Methods: The present study assessed a total of 32 patients who were classified as F3 (mood disorders) or F4 (neurotic disorders) according to the ICD-10, and who attended GCBT sessions offered at The Tokai University Hospital. Depression and mood in these patients were assessed before and after GCBT by using the Center for Epidemiologic Studies Depression Scale (CES-D) and the Profile of Mood States (POMS). Results: A comparison of pre- and post-GCBT CES-D scores among all patients as well as within F3 and F4 groups showed a significant decrease in CES-D scores after GCBT. As for the POMS, a comparison of pre- and post-GCBT scores among all patients showed a significant improvement in "vigor" and "confusion" scores after GCBT. Discussion: The present study demonstrated that GCBT can relieve depression and improve some mood states. Furthermore, the GCBT sessions offered proved to be effective even when administered to a group of participants consisting of both mood disorders and neurotic disorder patients.
\end{abstract}

Sawaguchi N et al. / Arch Clin Psychiatry. 2018;45(5):107-11

Keywords: Cognitive behavioral therapy, group therapy, mood disorders, neurotic disorders.

\section{Introduction}

In developed countries, such as England and the United States, a form of psychotherapy called Cognitive Behavioral Therapy (CBT) has well-established efficacy in the treatment of mood and anxiety disorders, and is recommended in guidelines for the management of these disorders ${ }^{1-3}$. Furthermore, previous studies carried out overseas have demonstrated that group cognitive-behavioral therapy (GCBT) is equally effective as one-on-one CBT sessions ${ }^{4,5}$.

In Japan, National Health Insurance (NHI) points have been assigned to the use of CBT in the treatment of mood disorders since April 2010. In addition, previous research on the effectiveness of CBT in patients with major depressive disorder found that patients experienced a drop in depression after 16 sessions of CBT ${ }^{6}$. In actual clinical settings, however, it is not practical for a psychiatrist to routinely administer CBT on a one-on-one basis. Some medical institutions therefore offer CBT in group settings. Although there are several published studies in Japan that address the effectiveness of GCBT in the treatment of psychiatric disorders, many of such studies focus only on mood disorders (including major depressive disorder) ${ }^{7-9}$. Furthermore, because there are no established criteria for GCBT, individual medical facilities create their own protocols and programs but the validity of such protocols and programs has not yet been verified. Although GCBT could be effective in treating not only major depressive disorder but also anxiety disorder and bipolar disorder (particularly, bipolar depression) ${ }^{10}$, no study has yet been conducted in Japan to evaluate the effectiveness of GCBT on psychiatric disorders other than major depression disorder.

Tokai University Hospital's outpatient psychiatric department has been offering GCBT as part of the day care rehabilitation program since September 2008, and has also been administering GCBT sessions on outpatient basis to patients with depression and anxiety disorders since June 2012. As is the case with many other medical institutions in Japan, the GCBT that we offer is based on modification of an individual CBT protocol. Ideally, the efficacy of such GCBT approaches should be verified by means of well-designed randomized controlled trials (RCTs). This, however, is impractical in actual clinical circumstances. Thus, we used an open study design to retrospectively examine how GCBT can alleviate depression and improve mood states based on the results of multiple psychological tests administered before and after GCBT.

\section{Subjects and methods}

\section{1) GCBT administered during the present study}

\section{(1) Staff members involved in GCBT and their roles}

GCBT staff consisted of one psychiatrist and three to four clinical psychologists. The lead clinical psychologist acted as facilitator of each session, with all the other clinical psychologists supporting and recording the work performed by the participants. The psychiatrist educated the participants about depressive and anxiety disorders symptoms and treatment from the psychological point of view during the first session, and collaborated afterwards with the clinical psychologists in providing the necessary support to the participants.

\section{(2) Inclusion criteria for GCBT}

Only the patients who routinely visited our outpatient psychiatric department for treatment and satisfied all of the following conditions were included in the present study:

- The patient is classified as F3 (mood disorders) or F4 (neurotic disorders) according to the International Statistical Classification of Diseases and Related Health Problems, 10 $0^{\text {th }}$ revision (ICD-10);

- The patient maintains a regular daily rhythm and is able to participate in eight GCBT sessions held once a week;

- The patient has presented good medication adherence;

- The patient has pledged to protect the personal information of other participants whom he/she will be encountering during the sessions; 
- The patient has agreed to take psychological tests before and after the course of therapy sessions, and to attend interviews during which he/she will be given feedback on the test results;

- The patient can work on and complete the assignments given to them during as well as between sessions;

- The patient is not at risk of self-harm or causing harm to others.

Patients were classified according to the primary diagnosis made at the time of enrollment in the study. This diagnosis, which was made by the psychiatrist with over five years of clinical experience, was based on the ICD-10 classification system provided by the World Health Organization (WHO). Patients having bipolar disorder and exhibiting no signs of mania were included in the $\mathrm{F} 3$ group because a previous study has demonstrated that CBT is also effective in preventing the recurrence of bipolar depression in patients with bipolar disorder ${ }^{10}$.

\section{(3) Overview of GCBT}

Figure 1 provides an overview of GCBT. Our GCBT protocol/ program is based on the individual CBT protocol developed by the Ministry of Health, Labor and Welfare in 2009. According to a previous study, a GCBT program generally consists of 8 to 69 hours of sessions conducted over a period of 5 weeks to 9 months 4 . Taking into consideration the factors associated with the financial situation and treatment period of the participants, we developed a GCBT program consisting of 8 sessions (a total of 12 hours). Because GCBT is administered to a closed group, no new members are admitted even when a group member drops out of the therapy. The GCBT we used in the present study was unique in that patients with mood disorders and those with neurotic disorders received a course of GCBT together as a group, instead of being divided into different groups by type of disorders. During the two-day period both before and after the course of treatment, the participants received several different psychological tests. After the tests, they attended interviews during which they were given feedback on test results. In addition, during the feedback interviews conducted before the course of GCBT, GCBT staff members reminded the participants of the purpose of participating in GCBT. During the feedback interviews conducted after the course of GCBT, staff members compared the test results before and after the course of GCBT and discussed with the participants their future goals of therapy. An overview of each session is provided below.

\section{Introductory session (session 1): Psychological education}

The psychiatrist gave a lecture on the symptoms and treatment of depression and anxiety disorders. Afterwards, the lead clinical psychologist explained the cognitive model to the participants. Specifically, he briefed the participants about the seven types of automatic thoughts and asked the participants to reflect on their patterns of automatic thoughts.

\section{Earlier sessions (sessions 2 to 5): Cognitive restructuring}

In sessions 2 and 3, a cognitive model was introduced, where the participants summarized the thoughts, emotions, somatic reactions, and immediate behaviors associated with their past experiences. Furthermore, we evaluated whether any thoughts associated with past events fit the description of the seven automatic thoughts discussed in session 1, and how such thoughts were correlated with emotions.

In sessions 4 and 5, we provided a detailed description of cognitive restructuring. In addition, we used the thought record ${ }^{11}$ in our attempts to encourage participants to restructure past events cognitively.

\section{Later sessions (sessions 6 to 8): Behavioral activation and assertion}

In session 6, we explained and helped patients develop an action plan for behavioral activation. During this session, the participants listed the activities that they wished to undertake every day during the next week and discussed what they could do to achieve their goals. As an assignment to be completed by the next session, the participants were asked to actually undertake the listed activities. In session 7 , we

Administer psychological tests before the course of GCBT sessions.
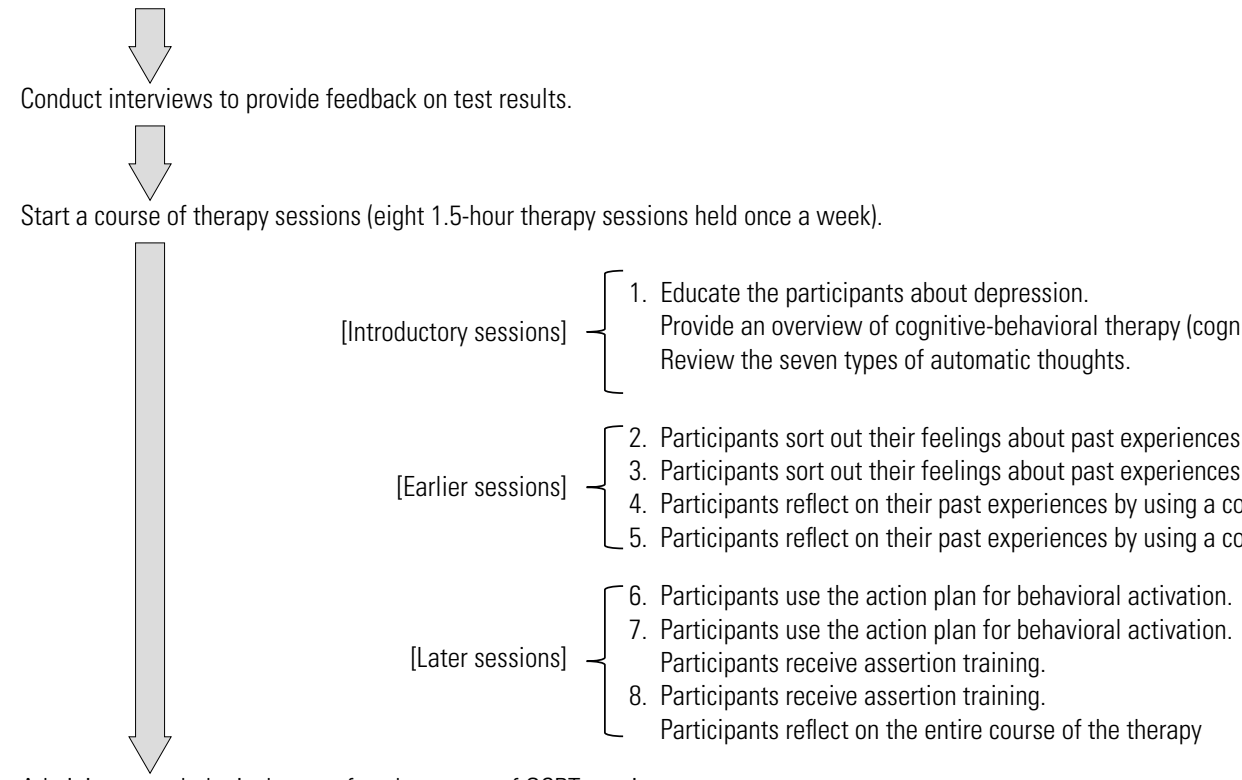

Administer psychological tests after the course of GCBT sessions.

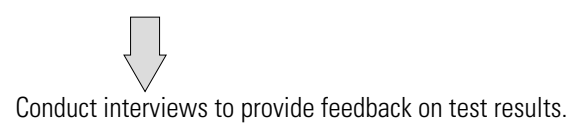

Figure 1. Overview of group cognitive-behavioral therapy. 
reviewed and reflected on the created action plans and explained the details about assertion training. In session 8 , the participants engaged in a role play of assertive behavior based on their past experiences. Then, the participants reflected on the entire course of the therapy.

\section{2) Effectiveness of GCBT}

There were 35 participants aged 18-62 years who not only met the inclusion criteria but also participated in GCBT during the period from June 5, 2012 to October 21, 2014. None of the participants with mood disorder exhibited psychotic features or had personality disorder. Three participants who dropped out of the study during one of the sessions were excluded from the analysis. Of these participants, 32 who attended all the sessions and who were also able to take the tests before and after the course of therapy sessions were included in the analyses.

Depression was evaluated as a primary outcome because, generally speaking, anxiety disorder patients no longer exhibit pathological anxiety by the time they are well enough to participate in the GCBT. The Center for Epidemiologic Studies Depression Scale (CES-D), which is accepted worldwide as a highly useful depression screening instrument, was used for the evaluation of depression. Furthermore, because some of the GCBT participants had anxiety disorder or bipolar disorder, the Profile of Mood Status (POMS) was used to measure mood state other than depression.

CES-D was used to measure depressive symptomatology. The CES-D is a questionnaire used to measure depressive symptoms experienced in the past week, consisting of 20 statements (e.g., "I was bothered by things that usually don't bother me", "My sleep was restless", etc.). The answers to each statement are given on a fourpoint rating scale: "none of the time", "1-2 days" " $3-4$ days", "5-7 days". A cut-off point of $15 / 16$ is used, with higher scores indicating greater symptoms of depression. In addition, the Profile of Mood Status (POMS) was used in a questionnaire survey that was carried out to assess the patients' mood states. The POMS is a questionnaire that contains 65 words/statements that describe feelings people have had during the past week. It assesses 6 mood subscales: "tension-anxiety (T-A)", "depression-dejection (D)", "anger-hostility (A-H)", "vigor (V)", "fatigue (F)", and "confusion (C)". Respondents rate themselves on a 5 -point scale ranging from 0 (not at all) to 4 (extremely). The cutoff point is 59/60 for all POMS subscales except "V", and 39/40 for "V".

The neuropsychiatric medication taken per day was calculated based on the equivalent of a standard medication: for antipsychotic medications, chlorpromazine was used as basis for calculation; for antidepressant, imipramine hydrochloride was used; for anxiolytics, diazepam was used ${ }^{12}$. Both surveys and neuropsychiatric medication were carried out before and after the course of GCBT sessions, as summarized in Figure 1. The pre-GCBT and post-GCBT scores from these surveys were compared to verify the effectiveness of GCBT.

\section{3) Data analysis}

Statistical analyses were performed. A Wilcoxon signed-rank test was used to compare pre-GCBT and post-GCBT CES-D and POMS scores, and a Mann-Whitney $U$ test was used to compare the test results among patients with different diagnoses. SPSS Statistics version 14.0 was used for these analyses.

The ethical aspects of the present study were reviewed and approved by the Institutional Review Board for Clinical Research, Tokai University.

\section{Results}

\section{1) Case profile}

Table 1 summarizes the baseline profiles of all cases. A total of 32 cases were included in the analyses, consisting of 18 male and 14 female patients, with the average age being $37.2(\mathrm{SD}=12.4)$. Based on the ICD-10, 18 of them were diagnosed by the psychiatrist as having mood disorders (F30-F39) (hereafter referred to as F3 group), and
14 of them were diagnosed as having neurotic disorders (F40-F48) (hereafter referred to as F4 group).

\section{2) CES-D}

Twenty-eight out of 32 participants scored higher than the cut-off point pre-GCBT and 20 scored higher than the cut-off post-GCBT. The comparison of pre-GCBT and post-GCBT CES-D scores of all 32 cases by means of Wilcoxon signed-rank test revealed significantly lower CES-D scores after the therapy, thus indicating that GCBT may be effective in alleviating depression $(Z=4.14, \mathrm{p}<0.01, \mathrm{r}=0.732)$. Similar findings were obtained even when the same comparison was made within F3 and F4 groups (F3: $Z=-3.25, \mathrm{p}<0.01, \mathrm{r}=0.770, \mathrm{~F} 4$ : $\mathrm{Z}=-2.54, \mathrm{p}<0.01, \mathrm{r}=0.688)$ (Table 2.1).

\section{3) POMS}

A Wilcoxon signed-rank test was used to compare pre-GCBT and post-GCBT POMS scores. Patients scored significantly higher on the "vigor" $(\mathrm{V})$ subscale and exhibited increased levels of vitality after GCBT $(Z=-2.01, \mathrm{p}<0.05)$. Furthermore, patients scored significantly lower on the "confusion" (C) subscale after GCBT, and they actually became less confused and were able to make decisions with greater ease $(Z=-2.17, \mathrm{p}<0.05)$. However, when the same comparison was made within F3 and F4 groups, no significant differences were observed on any of the POMS subscales (Table 2.1).

The comparison of pre-GCBT CES-D and POMS scores between $\mathrm{F} 3$ and $\mathrm{F} 4$ groups revealed no significant differences on any of either the CES-D items or POMS subscales. Similarly, the comparison of post GCBT test scores between F3 and F4 groups revealed no significant differences on any of either the CES-D items or POMS subscales.

Table 1. Profile of participants $(n=32)$

\begin{tabular}{|c|c|c|c|}
\hline Average age (S.D.) & & $37.2( \pm 12.4)$ & \\
\hline F/M & & $14 / 18$ & \\
\hline \multirow[t]{14}{*}{ ICD-10 } & F3 & F30 & 0 \\
\hline & & F31 & 2 \\
\hline & & F32 & 9 \\
\hline & & F33 & 3 \\
\hline & & F34 & 4 \\
\hline & & F38 & 0 \\
\hline & & F39 & 0 \\
\hline & F4 & F40 & 3 \\
\hline & & F41 & 6 \\
\hline & & F42 & 1 \\
\hline & & F43 & 3 \\
\hline & & F44 & 0 \\
\hline & & F45 & 1 \\
\hline & & F48 & 0 \\
\hline
\end{tabular}

\section{4) Neuropsychiatric medication}

There was no significant difference in the amount of neuropsychiatric medication taken per day in either group (Table 2.2).

\section{Discussion}

Although several studies have focused on the effectiveness of GCBT in alleviating depression, only a few studies have addressed how GCBT might help patients in other mood states. In the present study, we examined whether GCBT can alleviate depression and improve mood states by using the CES-D and the POMS, respectively. As a result, we (1) found a significant decrease in the depression scores calculated by using the CES-D after GCBT, (2) observed a significant 
Table 2.1. Comparison of CES-D/POMS score between pre- and post-CBT

\begin{tabular}{|c|c|c|c|c|c|c|c|c|c|c|c|c|c|c|c|c|c|}
\hline & \multicolumn{4}{|c|}{ Total $(n=32)$} & \multicolumn{4}{|c|}{$F 3(n=18)$} & \multicolumn{4}{|c|}{$F 4(n=14)$} & \multirow[b]{2}{*}{$\begin{array}{l}\text { a) vs } \\
\text { c) Z }\end{array}$} & \multirow[b]{2}{*}{$\begin{array}{c}\text { Effect } \\
\text { size } \\
\text { (r) }\end{array}$} & \multirow[b]{2}{*}{$\begin{array}{l}\text { b) vs } \\
\text { d) Z }\end{array}$} & \multirow[b]{2}{*}{\begin{tabular}{|c} 
Effect \\
size \\
$(\mathrm{r})$
\end{tabular}} \\
\hline & & Pre-CBT (SD) & Post-CBT (SD) & $Z$ & $\begin{array}{c}\text { Effect } \\
\text { size }(r)\end{array}$ & $\begin{array}{c}\text { Pre-CBT } \\
\text { a) (SD } \\
\text { values) }\end{array}$ & $\begin{array}{c}\text { Post- } \\
\text { CBT } \\
\text { b) (SD } \\
\text { values) }\end{array}$ & a) vs b) Z & $\begin{array}{l}\text { Effect } \\
\text { size }(r)\end{array}$ & $\begin{array}{c}\text { Pre-CBT } \\
\text { c) (SD } \\
\text { values) }\end{array}$ & $\begin{array}{c}\text { Post- } \\
\text { CBT } \\
\text { d) (SD } \\
\text { values) }\end{array}$ & c) vs d) Z & $\begin{array}{c}\text { Effect } \\
\text { size } \\
(r)\end{array}$ & & & & \\
\hline \multicolumn{2}{|c|}{ CES-D } & $25.97(10.55)$ & $20.25(10.01)$ & $-4.14^{* *}$ & 0.732 & 25.83 & 20.39 & $-3.25^{* *}$ & 0.770 & 26.14 & 20.07 & $-2.54^{* *}$ & 0.688 & 0.02 & 0.000 & 0.32 & 0.001 \\
\hline \multirow[t]{6}{*}{ POMS } & T-A & $60.43(12.36)$ & $57.72(13.87)$ & -1.29 & 0.229 & 60.44 & 56.89 & -1.07 & 0.257 & 60.43 & 58.79 & -0.56 & 0.148 & 0.10 & 0.000 & 0.15 & 0.001 \\
\hline & $D$ & 66.72 (13.52) & $64.22(13.13)$ & -1.40 & 0.248 & 67.89 & 63.83 & -1.94 & 0.402 & 65.21 & 64.71 & -0.04 & 0.000 & 0.53 & 0.002 & 0.25 & 0.001 \\
\hline & $\mathrm{A}-\mathrm{H}$ & $51.25(12.87)$ & $52.84(14.32)$ & -0.53 & 0.096 & 51.22 & 53.06 & -0.83 & 0.201 & 51.29 & 52.57 & -0.04 & 0.019 & 0.06 & 0.000 & 0.29 & 0.001 \\
\hline & V & $37.34(6.05)$ & $40.22(8.02)$ & $-2.01^{*}$ & 0.357 & 37.56 & 40.72 & -1.76 & 0.420 & 37.07 & 39.57 & -0.71 & 0.202 & 0.17 & 0.001 & \begin{tabular}{|l|}
1.07 \\
\end{tabular} & 0.004 \\
\hline & $\mathrm{F}$ & $61.34(12.74)$ & $59.53(13.72)$ & -1.01 & 0.18 & 61.67 & 60.61 & -0.23 & 0.061 & 60.93 & 58.14 & -1.61 & 0.436 & 0.29 & 0.001 & 0.53 & 0.002 \\
\hline & $C$ & $63.75(14.01)$ & $59.53(11.35)$ & $-2.17^{*}$ & 0.385 & 63.94 & 58.78 & -1.89 & 0.451 & 63.50 & 60.50 & -1.02 & 0.285 & 0.08 & 0.000 & 0.32 & 0.001 \\
\hline
\end{tabular}

CES-D: The Center for Epidemiologic Studies Depression Scale.

POMS: The Profile of Mood Status

T-A: Tension - Anxiety; D: Depression - Dejection; A-H: Anger - Hostility; V: Vigor; F: Fatigue; C: Confusion.

Total, a) vs b), and c) vs d) : Wilcoxon signed-rank test.

a) vs c) and b) vs d) : Mann-Whitney U test.

Effect size $(r)$ depends on correlation coeficient.

${ }^{* *} p<0.01{ }^{*} p<0.05$.

Table 2.2. Comparison of the usage of neuropsychiatric medication between pre- and post-CBT

\begin{tabular}{|c|c|c|c|c|c|c|c|c|c|c|c|c|c|c|c|}
\hline & \multicolumn{5}{|c|}{ Total } & \multicolumn{5}{|c|}{ F3 } & \multicolumn{5}{|c|}{ F4 } \\
\hline & $n$ & $\begin{array}{l}\text { Pre- } \\
\text { CBT } \\
\text { (SD) }\end{array}$ & $\begin{array}{c}\text { Post- } \\
\text { CBT } \\
\text { (SD) }\end{array}$ & $Z$ & $\begin{array}{l}\text { Effect } \\
\text { size }(r)\end{array}$ & $n$ & $\begin{array}{l}\text { Pre- } \\
\text { CBT } \\
\text { (SD) }\end{array}$ & $\begin{array}{c}\text { Post- } \\
\text { CBT } \\
\text { (SD) }\end{array}$ & $Z$ & $\begin{array}{l}\text { Effect } \\
\text { size (r) }\end{array}$ & $n$ & $\begin{array}{l}\text { Pre- } \\
\text { CBT } \\
\text { (SD) }\end{array}$ & $\begin{array}{c}\text { Post- } \\
\text { CBT } \\
\text { (SD) }\end{array}$ & $Z$ & $\begin{array}{l}\text { Effect } \\
\text { size }(r)\end{array}$ \\
\hline $\begin{array}{l}\text { Antidepressannt } \\
\text { (imipramine equivalent, mg/day) }\end{array}$ & 21 & $\begin{array}{c}123.2 \\
(95.27)\end{array}$ & $\begin{array}{c}98.8 \\
(84.01)\end{array}$ & 1.29 & 0.282 & 12 & $\begin{array}{c}100.0 \\
(58.77)\end{array}$ & $\begin{array}{c}101.0 \\
(106.1)\end{array}$ & 0.67 & 0.195 & 9 & $\begin{array}{c}154.2 \\
(126.71)\end{array}$ & $\begin{array}{c}95.8 \\
(46.35)\end{array}$ & 1.35 & 0.451 \\
\hline $\begin{array}{l}\text { Anxiolytics } \\
\text { (diazepam equivalent, mg/day) }\end{array}$ & 20 & $\begin{array}{c}14.1 \\
(12.39)\end{array}$ & $\begin{array}{c}15.6 \\
(19.93)\end{array}$ & 0.02 & 0.006 & 9 & $\begin{array}{c}17.9 \\
(16.87)\end{array}$ & $\begin{array}{c}22.7 \\
(27.13)\end{array}$ & 0.51 & 0.169 & 11 & $\begin{array}{c}11.0 \\
(6.30)\end{array}$ & $\begin{array}{c}9.8 \\
(9.11)\end{array}$ & 0.65 & 0.196 \\
\hline $\begin{array}{l}\text { Antipsychotic } \\
\text { (chlorpromazine equivalent, mg/day) }\end{array}$ & - & - & - & - & & 4 & $\begin{array}{c}156.4 \\
(200.29)\end{array}$ & $\begin{array}{c}50.2 \\
(99.88)\end{array}$ & 0.44 & 0.183 & - & - & - & - & - \\
\hline
\end{tabular}

Total, F3, F4: Wilcoxon signed-rank test effect size (r) depends on correlation coefficient.

increase in the "vigor" subscale scores and a significant decrease in the "confusion" subscale scores on the POMS after GCBT, and (3) confirmed that these effects (those mentioned in (1) and (2) above) can be produced even among a group of participants including not only mood disorder patients (F3) but also neurotic disorder patients (F4) who have been diagnosed by using the ICD-10.

These findings, along with similar results published overseas ${ }^{4,5}$ as well as in Japan ${ }^{7-9}$, suggest that GCBT could also be effective in alleviating depression. CBT is believed to be effective for patients with depression because it involves interventions aimed at addressing negative automatic thoughts that lead to depression. In fact, Mukhtar and Nishimura have reported negative automatic thoughts to be one of the predictor variables that is associated with the effectiveness of GCBT in alleviating depression ${ }^{13,14}$. In our GCBT sessions, we focused on replacing negative automatic thoughts with more adaptive thoughts by using a cognitive restructuring technique which is believed to have contributed to the relief of depression.

There are no studies that evaluated GCBT in patients with major depressive disorder or anxiety disorder and evaluated their effects using POMS.

An improvement in the "vigor" subscale scores after GCBT can be attributed to two reasons: (1) reflecting on past experiences through cognitive restructuring helps alleviate the participants' psychological burden, and (2) preparing and implementing an action plan help improve motivation and activeness in participants. A decrease in the "confusion" subscale scores after GCBT can also be attributed to two reasons: (1) exchanging ideas within group members helps each member discover feelings that he or she had not been aware of before, and (2) participants are able to sort out their feelings within a specific framework by using a cognitive restructuring technique. The same comparison (i.e. the comparison of pre- and post-GCBT "vigor" and "confusion" subscale scores) made within F3 and F4 groups also revealed similar trends, but the differences were not statistically significant. This may be due to the small sample size. On the T-A and F subscales, the participants scored higher than the cut-off pre- GCBT and lower than the cut-off post- GCBT, although no significant differences were found between pre- and post-GCBT subscale scores. Although this non-significance may be due to the small sample size, it could also suggest the limited effectiveness of our GCBT approach. On the other hand, although a significant difference was found between pre- and post-GCBT CES-D scores, no significant difference was found between pre- and post-GCBT "Depression/Anxiety" POMS subscale scores. This may be attributed to the fact that CES-D contains some statements that reflect everyday aspects of life, including appetite and sleep, which are not addressed by the POMS.

While most of the previous studies tested GCBT among only those patients diagnosed with mood disorders, the present study offered GCBT sessions to a group of participants consisting of not only those diagnosed with ICD-10 F3 mood disorders but also those diagnosed with ICD-10 F4 neurotic disorders. Interestingly, it demonstrated that GCBT is effective in alleviating depression and improving mood states even within a group of individuals diagnosed with different types of psychiatric disorders. Recently, the effectiveness of GCBT by a unified protocol covering both mood disorders and anxiety disorders has been reported, however our GCBT is simple and fewer than these unified protocols ${ }^{15}$. Moreover, our CBT was effective for both mood disorder and anxiety disorder, this finding suggests that our GCBT may be applied to a wider range of patients. A previous study reports that witnessing what other people say or do help participants gain greater self-insight into their own thoughts and behaviors ${ }^{16}$. Applying GCBT to a wider range of 
patients can benefit participating patients because they will then have a greater chance of encountering someone with similar backgrounds, with whom they can share their experience and from whom they can seek advice or reassurance.

The present study has, however, some limitations. Firstly, although CBT protocols targeting individual patients (such protocols are sometimes called "individual CBT protocols") are well-established in Japan, many healthcare providers in Japan are still unfamiliar with GCBT protocols. Both in terms of their design and the number of sessions held, the GCBT sessions provided in the present study are based on modification of an individual CBT protocol. We have not yet verified whether our GCBT protocol/program is equally effective as the individual CBT. Secondly, because the present study used a retrospective design and an open study design, the effects of drug therapy or other outpatient treatment and care received by the participants were not fully controlled for. Thus, in order to draw definitive conclusions about the treatment effect of our GCBT approach, we need to reevaluate our approach by using more rigorous research methods like RCTs. Thirdly, although the present study included patients belonging to the F4 category (i.e., patients with anxiety disorder, etc.), our analysis failed to specifically focus on changes in anxiety status. However, the F4 group on average scored 60.4 points on the T-A subscale of the POMS, which was only slightly higher than a cut-off value of 60 . Furthermore, no difference was observed between the F3 and F4 groups at baseline or at the end of GCBT. Fourthly, the follow-up period was not long enough either to determine long-term treatment effects of GCBT or to assess the possibility of recurrence. Finally the total sample size was limited to 32 patients. A larger sample size in future studies may render more significant results.

\section{Funding source}

This manuscript has no funding source.

\section{Disclosures}

Dr. Yano reports personal fees from Meiji Seika Pharma Co., Ltd.

Dr. Mikami received research support from Otsuka Pharmaceutical Co., Ltd., Shionogi \& Co., Ltd., Taisho Pharmaceutical Co., Ltd., honoraria from Otsuka Pharmaceutical Co., Ltd., Eli Lilly and Company, Shionogi \& Co., Ltd. and Shire Japan, and a consulting fee from Otsuka Pharmaceutical Co., Ltd.

Dr. Yamamoto reports grants from Esai Co., Ltd., grants and personal fees from Otsuka Pharmaceutical Co., Ltd., personal fees from Meiji Seika Pharma Co., Ltd., Sumitomo Dainippon Pharma Co., Ltd., Pfizer Japan Inc., Mitsubishi Tanabe Pharma Corporation, TEIJIN Pharma Limited, from Eli Lilly and Company, Yoshitomiyakuhin Corporation, Janssen Pharmaceutical K.K., Merck Sharp \& Dohme, GlaxoSmithKline K.K., Daiichi Sankyo Co., Ltd., EPS Holdings, Inc., and grants from a Health and Labor Sciences Research Grant, outside the submitted work.

Mr. Sawaguchi, Ms. Onaka, Ms. Hikima, and Mr. Kuroki have no disclosures.

\section{References}

1. National Institute for Health and Clinical Excellence (NICE). CG90 Depression in adults: recognition and management. 2009. Available from: https://www.nice.org.uk/guidance/cg90. Accessed on: Nov. 17, 2017.

2. National Institute for Health and Clinical Excellence (NICE). CG113 Generalized anxiety disorder and panic disorder in adults: management. 2011. Available from: https://www.nice.org.uk/guidance/cg113. Accessed on: Nov. 17, 2017.

3. American Psychiatric Association (APA). Practice guideline for the treatment of patients with major depressive disorder. $3 \mathrm{rd}$ ed. 2010. Available from: https://psychiatryonline.org/pb/assets/raw/ sitewide/practice_guidelines/guidelines/mdd.pdf. Accessed on: Nov. 17, 2017.

4. Oei TP, Dingle G. The effectiveness of group cognitive behaviour therapy for unipolar depressive disorders. J Affect Disord. 2008;107(1-3):5-21.

5. Scott MJ, Stradling SG. Group cognitive therapy for depression produces clinically significant reliable change in community-based settings. Behav Psychother. 1990;18:1-19.

6. Fujisawa D, Nakagawa A, Tajima M, Sado M, Kikuchi T, Hanaoka M, et al. Cognitive behavioral therapy for depression among adults in Japanese clinical settings: a single-group study. BMC Res Note. 2010;3:160.

7. Ito D, Kaneko Y, Suyama H, Kanaya T, Tanoue A, Koseki S, et al. Effects of Cognitive Behavioral Group Therapy on Adults with Depression: A Controlled Clinical Trial. Japan Assoc Behav Cogn Ther. 2012;38(3):169-79.

8. Matsunaga M, Suzuki S, Okamoto Y, Yoshimura S, Kunisato Y, Jinnin $\mathrm{R}$, et al. Cognitive Behavioral Group Therapy Conducted by Clinical Psychologists for Individuals with Major Depression: CBGT Program Conducted in a University Hospital. Japan Assoc Behav Cogn Ther. 2012;38(3):181-91.

9. Sato H, Tanno Y. The Effect of Cognitive behavioral therapy for depression delivered by Japanese psychologists: a systematic review. Japan Assoc Behav Cogn Ther. 2012;38(3):157-67.

10. Chiang KJ, Tsai JC, Liu D, Lin CH, Chiu HL, Chou KR. Efficacy of cognitive-behavioral therapy in patients with bipolar disorder: A metaanalysis of randomized controlled trials. PLoS One. 2017;12(5):e0176849.

11. Greenberger D, Padesky C. Mind over mood: change how you feel by changing the way you think. New York: The Guilford Press; 1995.

12. Kunikata H, Yoshinaga N, Nakajima K. Effect of cognitive behavioral group therapy for recovery of self-esteem on community-living individuals with mental illness: Non-randomized controlled trial. Psychiatry Clin Neurosci. 2016;70(10):457-68.

13. Mukhtar F, Oei TP. Predictors of Group Cognitive Behaviour Therapy outcomes for the treatment of depression in Malaysia. Asian J Psychiatr. 2011;4(2):125-8.

14. Nishimura $\mathrm{H}$. Effect of cognitive behavioral group therapy on depression and predictors of the effect response. J Japan Clin Psychol. 2016;34(2):141-50.

15. Barlow DH, Farchione TJ, Fairholme CP, Ellard KK, Boisseau CL, Allen LB, et al. Unified protocol for transdiagnostic treatment of emotional disorders: therapist guide. New York: Oxford University Press; 2011.

16. Nakashima M, Okumura Y. Cognitive-behavioral Group Therapy Practice Manual. Tokyo: Seiwa Shoten Publishers; 2011. 